\title{
METODOLOGÍA CIENTÍFICA EN VALORACIÓN Y SELECCIÓN AMBIENTAL
}

\author{
José María Moreno Jiménez \\ Juan Aguarón Joven \\ María Teresa Escobar Urmeneta \\ Facultad de Económicas \\ Universidad de Zaragoza - España \\ e-mail: moreno@posta.unizar.es
}

\section{Resumen}

El paradigma de la racionalidad sustantiva ha dominado la investigación científica durante la mayor parte del siglo XX. No obstante, cuando se trabaja con aspectos intangibles y múltiples criterios, como sucede en valoración y selección ambiental, esta filosofía positivista orientada a la predicción y el control presenta serias limitaciones, lo que ha llevado desde comienzo de los 70 a la búsqueda de nuevas aproximaciones. El constructivismo y la ciencia postnormal son algunas de las más extendidas en el campo de la economía ecológica. En lo que sigue, se analiza su relación con una de las aproximaciones más recientes en este ámbito: la racionalidad procedimental multicriterio, intentando establecer un marco para la metodología científica en selección ambiental.

Palabras clave: valoración ambiental, selección ambiental, metodología ambiental, negociación, caminos de consenso, racionalidad procedimental multicriterio. 


\section{Introducción}

La selección entre un conjunto discreto de "alternativas ambientales" está siendo uno de los problemas que más atractivo despierta, tanto desde un punto de vista teórico como práctico, en el campo ambiental. La consideración simultánea de múltiples criterios, habitualmente en conflicto, así como los problemas de valoración de aspectos intangibles y los de agregación en una escala válida para la toma de decisiones de las diferentes percepciones de la realidad que tienen los actores implicados en el proceso de resolución del problema, son algunas de las cuestiones que permanecen abiertas.

Tradicionalmente este tipo de problemas se resolvía siguiendo una aproximación economicista y simplista (normativa y axiomática) que, basada en unas hipótesis de racionalidad muy exigentes y poco realistas, aislaba el problema considerado en un "pequeño mundo" en el que habitualmente existían herramientas analíticas para su tratamiento. Este enfoque funcionalista, donde se separa la realidad "per se" de la forma en que ésta es percibida, supone dos hipótesis filosóficas asociadas a la descripción objetiva de la realidad (Buchanan et al., 1998): (1) la existencia de una realidad que describir (presunción ontológica), y (2) la posibilidad de alcanzar un conocimiento objetivo y universal de la misma, independiente de las valoraciones, emociones y puntos de vista personales (presunción epistemológica).

La primera hipótesis se refiere al mundo o realidad analizada (ontología) y la segunda, al conocimiento del mismo (epistemología). En cuanto al paradigma de racionalidad sustantiva, soporte de esta filosofía positivista, era y es el seguido por las ciencias formales y naturales (en muchos casos también por la economía). En él (Moreno, 1998) se mantenía el significado de Ciencia propuesto por los tres clásicos griegos (Sócrates, Platón y Aristóteles) que venía caracterizado por la existencia de una verdad única y la autonomía, entendida como la separación entre lo "racional" y lo "ético", o si se prefiere la separación entre lo objetivo y lo subjetivo.

Un estudio filosófico sobre la toma de decisiones subjetivas o cualitativas puede verse en Brugha (1998a,b,c), quien propone un sistema para analizar las decisiones cualitativas basado en cuestiones dicotómicas. Respuestas a preguntas relativas a qué, planificación ("planning") y ejecución ("putting"), y dónde, lugar ("place") y personas ("people"), dan lugar a las cuatro actividades consideradas como fases de un ciclo: proposición, percepción, influencia ("pull") y empuje ("push"). Contemplando una tercera dimensión, quién, con la dicotomía decisiones internas frente a externas, se tienen tres niveles de compromiso para las internas (somático, sicológico y neumático) y otros tres para las externas (técnico, otras personas y situación).

Empíricamente se ha comprobado que la aplicación de la racionalidad sustantiva (RS) en las ciencias sociales presenta numerosas limitaciones cuando se enfrenta a problemas en los que el subjetivismo, la incertidumbre y las interrelaciones entre los diferentes actores que intervienen en el proceso de toma de decisiones son fundamentales en su resolución. En la actualidad, la separación entre lo racional y lo ético está siendo abandonada en el campo ambiental. Más aún, los esfuerzos se están dirigiendo hacia la incorporación en los procesos de decisión de las visiones de la realidad de todos los actores participantes (aspectos subjetivos y cualitativos), incluyendo en ellas los aspectos sociales, éticos y culturales.

En la última década se han presentado diversas aproximaciones y metodologías "pragmáticas" o "constructivistas" que han orientado los estudios de selección ambiental hacia el análisis de los propios procesos de decisión. Checkland \& Scholes (1990) introducen el concepto de 
ciencia flexible o blanda ("soft system science"). Funtowicz \& Ravetz $(1991,1994)$ el de ciencia postnormal. Harvey (1989) y Midmore (1996) el de postmodernismo, y Gandy (1996) el de realismo crítico. Los puntos fuertes y débiles de estas aproximaciones, así como sus dependencias, son estudiados por Woodhill \& Roling (1999). Tacconi (1998) sugiere la complementariedad entre las metodologías constructivistas y la ciencia postnormal.

A continuación se analiza la relación existente entre la ciencia postnormal, el constructivismo y el paradigma de la racionalidad procedimental multicriterio (RPM), la propuesta metodológica sugerida (Moreno, 1996, 1997; Moreno et al., 1999). La estructura del trabajo ha quedado como sigue: La sección 2 presenta las características de la RS y los diferentes procedimientos empleados en valoración ambiental. La sección 3 incluye las características de las tres aproximaciones consideradas: la ciencia postnormal, el constructivismo y la RPM. La sección 4 destaca una serie de aspectos que deben contemplar las metodologías seguidas en selección ambiental y por último, la sección 5 recoge las conclusiones más importantes.

\section{Enfoque Tradicional. Limitaciones}

Aparte del interés despertado en los últimos años por los temas ambientales, desde que el tratado de Maastrich adoptó el principio de sostenibilidad, se ha hecho necesario, al menos dentro de la Comunidad Europea, que todo trabajo de valoración y selección ambiental integre los conceptos ambientales en los procesos de toma de decisiones (Mirasgedis \& Diakoulaki, 1997). El enfoque tradicional en selección ambiental está basado en valoraciones económicas cuyo soporte metodológico sigue el paradigma de racionalidad sustantiva.

El concepto de racionalidad es uno de los más tratados en la literatura científica (véase Hargreaves Heap et al., 1992), de ahí que existan numerosas definiciones e interpretaciones. Simon $(1964,1972)$ distingue entre racionalidad, en su sentido más amplio, entendida como la adaptación de los medios disponibles a un fin, y la racionalidad seguida por las diferentes teorías y modelos considerados en la toma de decisiones. En este caso, incluye la racionalidad sustantiva y la procedimental.

\subsection{Racionalidad Sustantiva y Procedimental}

La racionalidad sustantiva (ideal, estricta o instrumental) corresponde al enfoque tradicional, ortodoxo o clásico que ha dominado el campo de la toma de decisiones hasta los años 70 . Está orientada a la salida, y supone que se pueden predecir las consecuencias, o salidas asociadas a las alternativas. Tiene un carácter normativo, e indica qué hacer y cuándo hacerlo.

Las hipótesis de racionalidad que exige no afectan a la forma en la que las decisiones son tomadas, se refirieren exclusivamente a los resultados asociados a las elecciones realizadas. Guía la elección de una acción (la mejor entendida como la óptima) en un problema dado. Tiende a trabajar suponiendo: un único criterio (objetivo), el carácter estático de las preferencias consideradas en el proceso de decisión y su especificación a priori (permanecen fijas).

Es de gran belleza formal, contenido lógico (coherente), y básicamente objetiva. Suele ser eficaz en la resolución de problemas altamente estructurados, planteados en los pequeños mundos. Persigue la predicción y el control (enfoque duro), y presenta notables limitaciones en situaciones reales caracterizadas por un alto grado de incertidumbre (ilegitimada por la realidad social). 
La racionalidad procedimental (descriptiva) corresponde al enfoque moderno en toma de decisiones, surgido a comienzo de los 70. Está orientada al proceso, y supone que un mejor conocimiento del mismo, permite mejorar las salidas. Tiene un carácter descriptivo, e indica cómo funciona el sistema.

Las hipótesis de racionalidad sí afectan a la forma en que se toman las decisiones, más aún, se refieren fundamentalmente al proceso de toma de decisiones propiamente dicho. Guía la elección de la investigación completa. El procedimiento que alcanza la solución es óptimo. Tiende a trabajar suponiendo múltiples criterios (objetivos), contempla el carácter dinámico de las preferencias y su reformulación a lo largo del proceso de resolución, fruto del aprendizaje que se produce.

Es muy flexible y adaptativa, permitiendo la incorporación de lo objetivo y lo subjetivo. Suele ser efectiva en la resolución de problemas poco estructurados (complejos), planteados en el gran mundo. Persigue la comprensión y el consenso (enfoque blando). Se comporta acertadamente en situaciones reales con un alto grado de incertidumbre.

Junto a las dos escuelas mayoritariamente seguidas durante el último cuarto del siglo XX en la toma de decisiones: la normativa (Von Neumann \& Morgestern, 1944; Savage, 1954; Fishburn, 1970), basada en el paradigma de racionalidad sustantiva, que indica cómo deberían tomarse las decisiones y qué métodos utilizar para ello, y la descriptiva, (Lichtenstein \& Slovic, 1971; Simon, 1972; Tversky \& Kanheman, 1972; Kanheman \& Tversky, 1979), basada en el paradigma de racionalidad procedimental, que indica cómo se toman las decisiones, en la última década se está planteando la existencia de una tercera vía, la escuela prescriptiva, constructivista o clínica, que basada en nuevos paradigmas de racionalidad (Tversky, 1988; Howard, 1992; Keeney, 1992, 1994; Saaty, 1994; Moreno, 1996, etc.) busca mejorar los procesos de decisión. Esta nueva aproximación, así como las más recientes propuestas en este sentido, pueden verse en la tercera sección.

\subsection{Valoración Ambiental}

A continuación se mencionan los procedimientos de valoración ambiental habitualmente utilizados en la literatura. Conforme a la tendencia mayoritariamente seguida en nuestros días, la finalidad de los diferentes procedimientos usados en selección ambiental es fundamentalmente la preservación del entorno. Para ello, se debe: (1) reconocer e incorporar en los modelos asociados a los diferentes enfoques analíticos empleados en la resolución de problemas, los efectos o impactos ambientales; y (2) valorar las consecuencias de los mismos en una escala de medida apropiada para efectuar la priorización y selección final.

Los métodos de evaluación más utilizados en la literatura suelen agruparse en dos grandes bloques: (i) los basados en valoraciones económicas y (ii) los basados en valoraciones no económicas. Los primeros internalizan los recursos naturales en el sistema productivo asignando un precio o valor monetario, tanto a los beneficios como a los costes ambientales. Este precio no es exactamente la valoración económica del impacto, sino una cantidad o punto de referencia que permite comparaciones entre alternativas. Los segundos, donde se incluyen numerosas y muy diversas aproximaciones, proporcionan una visión más realista del problema integrando las valoraciones de las consecuencias efectuadas en unidades de "impacto ambiental", en una escala apropiada para la elección de la "mejor" alternativa. Al igual que en las valoraciones económicas, el significado de los números obtenidos en los métodos de evaluación no económicos es muy discutible. 
Las distintas aproximaciones seguidas en la valoración económica del medio ambiente pueden estructurarse (Blasco et al., 1994; Moreno, 1998) conforme a la medición directa o indirecta de los efectos, a su comportamiento real o supuesto y a la referencia o no al mercado, en: métodos directos e indirectos totales, y métodos directos e indirectos de referencia al mercado. La ausencia de referencia al mercado de los dos primeros métodos (totales) ha favorecido su abandono en favor de los dos últimos.

Los métodos indirectos de referencia al mercado evalúan los costes provocados por el disfrute de los bienes ambientales. Entre estos métodos destacan el Método de Coste del Viaje o Desplazamiento (Clawson \& Knetsch, 1966) y el Método de Variables o Precios Hedónicos (Griliches, 1971; Rosen, 1974). Los métodos directos con mercados hipotéticos generan un mercado artificial para obtener la disposición al pago o a la compensación incluyendo la valoración de los no usuarios. En este caso, destaca el Método de Valoración Contingente (Ciriacy-Wantrup, 1952; Davis, 1963).

Respecto a las debilidades de estas aproximaciones, mencionar que los modelos de referencia indirecta al mercado no evalúan los precios en competencia perfecta, coexistiendo precios políticos, monopolios, impuestos y subvenciones, y que los métodos directos, como le ocurre al de valoración contingente, generan un mercado de un bien, con frecuencia desconocido y habitualmente sin coste. En este caso, el método de estimación es poco fiable por la gran dependencia del contexto donde se produce el estudio. En general, estos procedimientos economicistas presentan serias limitaciones, en especial: las relativas a la fijación de los actores implicados, lo que influye en los valores considerados (uso, opción y existencia); la inexistencia de un mercado ambiental y la consideración de aspectos intangibles difícilmente cuantificables en términos económicos.

Por otro lado, el economicista enfoque tradicional está basado en el paradigma de la racionalidad sustantiva cuyas hipótesis restrictivas (Moreno, 1996, 1997; Moreno et al., 1999) no lo hacen el más apropiado en la priorización y selección ambiental. El comportamiento optimizador (maximización del bienestar) de la escuela tradicional, utilitarista u ortodoxa, requiere el conocimiento previo de las alternativas, de sus consecuencias y del criterio seguido para la evaluación y comparación de las mismas. En la práctica, el decisor no conoce las consecuencias de las alternativas, sino las expectativas de las mismas. Además, existe más de un objetivo y no tiene capacidad ilimitada para producir información, por lo que requiere la utilización de paradigmas de racionalidad menos estrictos donde las decisiones se tomen en un tiempo limitado y basándose en una información parcial.

Algunas características de los problemas ambientales (Janssen, 1992; Moreno, 1996) como su complejidad (múltiples actores, criterios, escenarios, etc.), incertidumbre (ausencia de modelos subyacentes e información cierta, lagunas en el conocimiento, etc.), irreversibilidad (existencia de efectos irreversibles con poca verosimilitud), equidad intergeneracional (consideración de las generaciones futuras), y el subjetivismo asociado a lo trascendental, comprensión y descripción (Buchanan et al., 1998), sugieren su resolución utilizando otros enfoques que siendo más abiertos, flexibles y realistas que la racionalidad sustantiva, se orienten fundamentalmente al aprendizaje, la comprensión y la comunicación (Moreno et al., 1999).

En este sentido se han propuesto en la literatura otras aproximaciones no monetarias en valoración ambiental entre las que destacan: a) los métodos de evaluación de impacto ambiental (EIA) y b) las técnicas de decisión multicriterio (TDM). Los métodos EIA proporcionan un valor asociado a cada alternativa que mida los efectos ambientales en las denominadas unidades de impacto ambiental. Los valores asignados son mera referencia 
numérica en operaciones calculistas, pero no tienen ningún significado intrínseco. Dentro de este grupo se incluyen la Matriz de Leopold (Leopold et al., 1971), el Método BatelleColumbus (Batelle-Columbus, 1972), el Método de Gómez Orea (Gómez Orea, 1988, 1992).

Las TDM son un conjunto de herramientas y procedimientos utilizados en la resolución de problemas de decisión complejos en los que intervienen diferentes actores y criterios. Estas técnicas pueden clasificarse conforme a numerosos criterios. En general, suelen seguir diferentes enfoques metodológicos entre los que destacan: 1) la consideración simultánea de todos los criterios (generación de soluciones eficientes); 2) la utilización de funciones valor o utilidad en las que se agregan la contribución de los distintos criterios; y 3) el empleo de otras aproximaciones como pueden ser la minimización de funciones distancia a un punto de referencia (ideal o meta), y la búsqueda de soluciones satisfactorias.

En el caso de decisión multicriterio discreta, la habitualmente empleada en selección ambiental, las tres aproximaciones más extendidas son: a) la Teoría de Utilidad Multiatributo (MAUT) de Keeney \& Raiffa (1976); 2) el Proceso Analítico Jerárquico (AHP) de Saaty $(1977,1980)$ y 3 ) los Métodos de Superación (MS) de la escuela francesa (Roy, 1985). Un estudio más detallado de las tres técnicas y de las aplicaciones ambientales de las mismas puede verse en Moreno (1998).

\section{Nuevas Aproximaciones}

Los procedimientos no monetarios (EIA, TDM) vistos en la sección anterior, requieren otros paradigmas decisionales más acordes con la filosofía actualmente existente en la búsqueda de la mejor decisión ambiental. Cuando se habla de la aplicación del método científico en la toma de decisiones (decisiones analíticas frente a intuitivas), se necesita establecer una serie de principios que permitan: organizar el pensamiento, estructurar el proceso mental interno, analizar el problema, sintetizar datos, información y conocimiento, y dotar de rigor metodológico, accesibilidad y publicidad a los resultados obtenidos (Roy, 1993).

Todo ello exige combinar aspectos tangibles e intangibles en una escala válida para la toma de decisiones, siguiendo una nueva racionalidad que sea (Saaty, 1996): a) simple en su construcción; b) adaptable a decisiones en grupo e individuales; c) acorde con nuestras intuiciones, valores y pensamientos; d) que potencie el compromiso y el consenso y e) que no exija una especialización suprema para su comprensión.

Antes de pasar a exponer las tres aproximaciones que se van a analizar en este trabajo, se establecerá la acepción dada a tres términos básicos en cualquier propuesta: paradigma, metodología y racionalidad.

En general, se entiende por paradigma el conjunto de conceptos, valores, hipótesis y procedimientos que proporcionan unidad a una disciplina científica. Aquí se considera como el conjunto de creencias o visiones del mundo en torno a las cuales se organiza la realidad (Kuhn, 1970; Berman, 1981; Lincoln \& Guba, 1985). Todo paradigma investigador puede definirse concretando su ontología (naturaleza de la realidad que el paradigma postula), su epistemología (naturaleza de las relaciones entre el conocedor y lo conocido) y su metodología (método de investigación seguido).

En sentido estricto, el término metodología se ha usado para referirse al estudio del proceso a través del cual las teorías se forman y justifican (Blaug, 1992). En un sentido más amplio, el significado de metodología se refiere al proceso de aprendizaje sobre lo social y natural del mundo (Hausman, 1992). 
Respecto al tercer término mencionado, en general (Simon, 1960), se entiende por racionalidad la adaptación de los medios a un fin, aunque nuestra acepción está más en la línea de Russell (1993) quien la considera como la elección de acuerdo con unas preferencias reveladas por el comportamiento del decisor. Las distintas definiciones de racionalidad (véase Hargreaves Heap et al., 1992) han dado lugar a las diferentes escuelas de pensamiento o aproximaciones seguidas en la toma de decisiones: racionalidad sustantiva (decisor racional); racionalidad limitada (decisor satisfactorio); racionalidad procedimental (decisor descriptivo); racionalidad constructiva (decisor pragmático); y racionalidad procedimental multicriterio (decisor cognitivo).

Conforme a los tres elementos que concretan un paradigma (ontología, epistemología y metodología), el de la racionalidad sustantiva, filosofía positivista o empirismo lógico, viene caracterizado (Tacconi, 1998) por:

- Ontología: Realista. La realidad está ahí y viene dada por leyes naturales inmutables en el tiempo. Estas leyes suelen buscar relaciones causa-efecto.

- Epistemología: Dualista/Objetivista. Las dos son posibles, siendo esencial para la investigación adoptar una postura distante y no interactiva.

- Metodología: Manipulación experimental. Las preguntas e hipótesis se establecen con antelación y están sujetas a contrastes empíricos en condiciones controladas.

El rechazo del positivismo por los filósofos de la ciencia a comienzos de los 70 (Suppe, 1974; Medawar, 1974), debido fundamentalmente a la dependencia entre las teorías y las valoraciones de los actores (Beed, 1992; Nelson, 1996) se ha ido incorporando lentamente al campo de las ciencias sociales. La ausencia de una realidad objetiva ha favorecido la aparición de dos propuestas alternativas: la posición de la realidad construida (Hesse, 1980) y la posición de la realidad creada (Lincoln \& Guba, 1985). La primera plantea que la realidad es una construcción de nuestras mentes, de ahí que al existir numerosos actores se tendrán numerosas realidades. La segunda plantea una visión más radical de la realidad que puede identificarse con el trabajo de los físicos. Afirma que la realidad no existe. Sólo interviene cuando es percibida.

Todas estas ideas resaltan el papel que en la metodología seguida en la toma de decisiones ambientales tienen los actores y sus visiones de la realidad, influidas decisivamente por los aspectos éticos y culturales. Es por tanto imprescindible incorporar en la metodología científica lo subjetivo e intangible, todo ello de alguna forma que garantice el rigor científico de cada una de las etapas seguidas en los procesos de resolución. Las características tradicionales del método científico, racionalidad y objetividad, están siendo reemplazadas por las de rigor, accesibilidad y publicidad.

Poéticamente, se puede decir que se ha pasado del concepto de Ciencia entendida como la "Iglesia de la Razón" o la "Religión de la Objetividad", al de "Templo de la Sabiduría" o "Meca del Conocimiento". En este sentido, se han formulado nuevas aproximaciones, como son:

\subsection{Ciencia postnormal en medio ambiente}

Entendiendo por ciencia normal el método científico (Kuhn, 1970) mayoritariamente seguido en la toma de decisiones. Funtowicz \& Ravetz $(1991,1994)$ plantean la ciencia postnormal. En esencia, es una adecuación de la ciencia normal a los nuevos tiempos en los que tanto los valores culturales y éticos, como la incertidumbre e ignorancia, deben ser contemplados expresamente e incorporados en los procesos de resolución. La demarcación entre las dos 
ciencias en un problema concreto se basa en dos factores: a) la intensidad de la incertidumbre y b) la intensidad de los aspectos decisionales (costos, beneficios y compromisos). Funtowicz \& Ravetz consideran tres niveles para la incertidumbre: técnico, metodológico y epistemológico, cuyas intensidades son menores en las ciencias normales que en las postnormales.

La ciencia postnormal se orienta a la gestión de la incertidumbre y a la mejora de los procesos mediante un diálogo interactivo y no un método deductivo. Este enfoque no es indiferente o neutro a los aspectos más intangibles (éticos, sociales y culturales). Para mejorar los procesos de decisión proponen: 1) prestar atención a la calidad de la investigación científica, en particular a los aspectos investigados (cómo se utilizan las técnicas y no si funcionan o no);2) incorporar al proceso de resolución a los actores o participantes; 3) incluir "periodismo de investigación" y técnicas relacionadas como formas de incorporar conocimiento en la discusión pública; y 4) intentar llegar a un compromiso de resolución de los problemas entre las partes implicadas.

Las caracterísitcas de su paradigma, en cuanto a ontología, epistemología y metodología, coinciden básicamente con las del constructivismo que se verá a continuación.

\subsection{Constructivismo}

Se ha desarrollado durante las últimas tres décadas (Geertz, 1973; Lincoln \& Guba, 1985; Hannigan, 1995; Weber, 1968; Maturana \& Varela, 1992) fruto de la convergencia de diferentes disciplinas (sicología, educación, sociología, ecología, etc.). Guba (ver Tacconi, 1998) resume las características del paradigma constructivista en:

- Ontología: Relativista. La realidad existe en la forma de múltiples construcciones mentales que dependen de las personas implicadas.

- Epistemología: Subjetivista. El investigador y lo investigado forman una entidad única. Los descubrimientos son el proceso de una interacción entre los dos.

- Metodología: Hermenéutica y dialéctica. Las construcciones son definidas hermenéuticamente (reglas para alcanzar la verdad) y comparadas y contrastadas dialécticamente con la ayuda de una construcción para la que existe consenso.

El paradigma constructivista es criticado por algunos ecologistas porque niegan las restricciones biofísicas de la vida social (Jacobs, 1996). Además, hay problemas al definir y medir la sostenibilidad, e incluso al formular las preguntas. Tres factores condicionan la investigación social objetiva: reactividad, indeterminación e interacción (Tacconi, 1998). Metodológicamente, si el positivismo se basa en leyes que soportan teorías cuyas hipótesis son contrastadas mediante métodos deductivos, el constructivismo propone un procedimiento alternativo: las hipótesis "trabajadas" (Cronbach, 1975). Esta aproximación (Lincoln \& Guba, 1985) viene caracterizada porque las realidades no pueden entenderse aisladas del contexto, y porque su conocimiento permite determinar si los logros pueden extenderse a otras situaciones. En ella, los métodos cualitativos son más empleados que los cuantitativos, y el análisis inductivo de datos es preferido al deductivo.

\subsection{Racionalidad Procedimental Multicriterio (RPM)}

Este paradigma (Moreno, 1996, 1997; Moreno et al., 1999) combina el soporte metodológico de la racionalidad procedimental con el potencial operativo y calculista de las técnicas de decisión multicriterio, y pretende adecuar el enfoque seguido en la resolución del problema, 
con las nuevas tendencias en la toma de decisiones, en general, y con el significado de la "mejor solución", en particular.

Este nuevo enfoque, de carácter descriptivo, adaptativo, cognitivo, negociador, sistémico y general, trata de ayudar en la toma de decisiones mediante un mejor conocimiento de su proceso de decisión, esto es, un mejor conocimiento de las etapas, escenarios, elementos, factores, interdependencias, actores, interrelaciones y procedimientos que incluye. En esencia, busca mejorar la calidad integral del proceso de toma de decisiones seguido por el sistema considerado. Para ello, intenta dotar de rigor científico cada una de las etapas y fases seguidas en el proceso de resolución. De esta forma se mejorará la efectividad, la eficacia, y la eficiencia del mismo evitando algunas de las "miopías" que numerosos estudios económicos presentan. Se entiende por efectividad, la fijación de los objetivos (criterios) apropiados para la resolución del problema; por eficacia, la consecución de los objetivos (metas) marcados; y por eficiencia, el lograr esos objetivos (económicos) con la mejor utilización posible de los recursos o inputs.

La búsqueda de una mayor calidad integral del proceso de resolución en vez de la búsqueda de la solución óptima del problema, responde a la nueva interpretación que se le está dando al concepto de la mejor solución. Este cambio de mentalidad está siendo recomendado, especialmente, en aquellos casos, como sucede en los problemas de selección globales, en los que lo conocido es mucho menor que lo desconocido. Cuando la incertidumbre y complejidad del problema tratado condicionan decisivamente los resultados obtenidos en la resolución del mismo, es preferible un estudio descriptivo en vez de uno normativo fuertemente dependiente del contexto.

En resumen, el análisis se dirigirá hacia: (1) la comprensión del proceso de decisión seguido; (2) el aumento del valor añadido del conocimiento alcanzado en la resolución del problema, esto es, la mejoría del conocimiento de las diferentes etapas, factores, elementos y actores, profundizando en el aprendizaje y justificación del mismo; (3) la detección de oportunidades de decisión que faciliten la formulación de nuevas alternativas; (4) el descubrimiento de las preferencias y gustos de los actores implicados, necesario en la fase de retroalimentación y (5) la potenciación del proceso de negociación y diálogo.

Conforme a los tres factores considerados en la especificación de todo paradigma (ontología, epistemología y metodología), el paradigma de la racionalidad procedimental multicriterio viene determinado por:

- Ontología: Relativista y emocional. La realidad viene dada por las diferentes visiones de la misma que poseen los actores implicados en los procesos de resolución. Se incorpora lo racional junto a lo emocional, incidiendo especialmente en aspectos hasta la fecha intangibles y no contemplados (culturales, sociales, éticos).

- Epistemología: Adaptativa. La incorporación de los deseos y creencias de los actores implicados en la resolución del problema, así como la continua modificación de sus necesidades y estructuras de preferencia, fruto del propio proceso de resolución, requiere una interacción entre los participantes y los conocimientos sobre contenidos y procedimientos aportados por los mismos.

- Metodología: Constructivismo cognitivo. La metodología persigue la detección de oportunidades de decisión, pautas de comportamiento o tendencias que puedan servir como punto de partida en los procesos de negociación y diálogo entre los implicados en la resolución del problema. Todo ello garantizando el rigor científico exigido a cualquier 
aproximación seguida en este campo. Recordemos que la búsqueda del consenso entre los actores del proceso de toma de decisiones es una de las pocas líneas de actuación universalmente reconocida como válidas en estudios ambientales.

La existencia de múltiples escenarios, criterios y actores lleva a la consideración conjunta de aspectos tangibles e intangibles, de valores numéricos y simbólicos, de procedimientos analíticos y simbólicos, lo que complica notablemente la validez de la metodología empleada en selección ambiental.

La Racionalidad Procedimental Multicriterio (constructivismo cognitivo) responde a las "características" exigidas en los últimos tiempos a la aplicación del método científico: rigor, accesibilidad y publicidad (Roy, 1993; Moreno, 1996). Consta de los siguientes pasos (Moreno, 1996, 1997): P1: Formulación y Descripción; P2: Modelización; P3: Incorporación de las preferencias. Emisión de juicios; P4: Priorización, Agregación y Síntesis; P5: Incertidumbre, Robustez y Retroalimentación y P6: Explotación del modelo: Aprendizaje y Negociación, y aunque traslada las ideas anteriores a todas las etapas consideradas en el proceso de toma de decisiones, incide fundamentalmente en la inicial y final del mismo, esto es, las dedicadas al planteamiento del problema (para evitar el error Tipo 3) y a la explotación del modelo (para lograr un solución efectiva).

\begin{tabular}{|c|c|c|c|}
\hline PARADIGMA & ONTOLOGÍA & EPISTEMOLOGÍA & METODOLOGÍA \\
\hline $\begin{array}{c}\text { Racionalidad } \\
\text { Sustantiva }\end{array}$ & Realista & Dualista/Objetivista & $\begin{array}{c}\text { Manipulación } \\
\text { Experimental }\end{array}$ \\
\hline Constructivismo & Relativista & Subjetivista & $\begin{array}{c}\text { Hermenéutica y } \\
\text { Dialéctica }\end{array}$ \\
\hline $\begin{array}{c}\text { Racionalidad } \\
\text { Procedimental } \\
\text { Multicriterio }\end{array}$ & $\begin{array}{c}\text { Relativista y } \\
\text { Emocional }\end{array}$ & Adaptativa & $\begin{array}{c}\text { Constructivismo } \\
\text { Cognitivo }\end{array}$ \\
\hline
\end{tabular}

\section{Bases para una Metodología en Selección Ambiental}

En lo que sigue se presentan una serie de recomendaciones, hechos estilizados, regularidades o patrones (Pulido, 1998) que deben tenerse presentes cuando se quiere emplear una metodología efectiva y valida, desde el punto de vista científico, en selección ambiental.

a) Los problemas ambientales se plantean habitualmente en macroentornos o grandes mundos (muy poco estructurados) en los que se integran numerosos microentornos o pequeños mundos (bastante estructurados).

b) La existencia de múltiples actores, entre ellos las generaciones futuras, exige la incorporación en los modelos de las diferentes visiones de la realidad (necesidades, preferencias y valoraciones) que cada uno aporta. Estas visiones, correspondientes al punto de vista de los actores "inmediatos", "próximos" y "lejanos", dependen del contexto en el que cada participante se encuentre (ámbito espacial y temporal) y de aspectos subjetivos estrechamente relacionados con los mismos (culturales, sociales, económicos, ambientales, éticos,...). 
c) La fijación de los actores implicados, con sus diferentes grados de participación, condicionan las valoraciones dadas a los efectos ambientales considerados (valor de uso, opción, existencia y valor expectante). Además, estas valoraciones no son estáticas en el tiempo pues las necesidades y preferencias de los actores varían continuamente, siendo aconsejable una metodología adaptativa, flexible y con retroalimentación (cognitiva y operativa).

d) Los problemas ambientales vienen caracterizados por una gran complejidad debida fundamentalmente a: el desconocimiento de los verdaderos aspectos relevantes del problema; la ambigüedad intrínseca de muchos de ellos y a la incertidumbre asociada a los mismos; la existencia de sucesos muy poco verosímiles pero con efectos irreversibles; la consideración de diversos actores, incluyendo las generaciones futuras, con sus respectivas descripciones, comprensiones y fijaciones de los aspectos relevantes; y la existencia de múltiples criterios, escenarios, factores e interrelaciones.

e) La forma de modelizar todos estos escenarios, factores, criterios, actores, interrelaciones y horizontes temporales y espaciales va a resultar determinante a la hora de seleccionar la metodología apropiada para la resolución del problema. La racionalidad procedimental multicriterio estructura el problema en dos bloques: el primero, en el que se recoge la parte menos ambigua y más conocida del problema (criterios, subcriterios, atributos y alternativas), se modeliza mediante una jerarquía (se supone independencia entre los elementos) y el segundo, en el que recoge la parte más abierta y menos estructurada del mismo, se modeliza mediante una red (se permite dependencia entre los elementos).

f) La combinación de datos objetivos junto a opiniones subjetivas (valores numéricos y simbólicos), requiere la utilización de escalas de medida que permitan integrar las valoraciones asignadas a los mismos en una escala válida para la toma de decisiones (seleccionar la "mejor" alternativa). En esencia se busca mejorar la calidad integral del proceso de toma de decisiones, mejorando el conocimiento que se tiene del mismo.

g) La imprecisión de los datos empleados en situaciones tan complejas y la ambigüedad de algunas situaciones, desautorizan las conclusiones obtenidas a partir de unos valores fijos para los parámetros. En este caso, más importante que la obtención de la solución óptima del problema es el aprendizaje y la comprensión derivados de la explotación del modelo.

h) Por otro lado, hay un problema adicional cuando se trabaja en selección ambiental. Los datos empleados en la misma suelen ser valores residuales provenientes de estudios previos. En este sentido, se sugiere trabajar con la información y el conocimiento obtenidos dentro de los propios procesos de resolución. Se entiende (Moreno \& Mata, 1992) por Dato un conjunto de números o símbolos no estructurados que vienen dados en bruto y sin dirigir; por Información a los datos dotados de estructura y finalidad, y por Conocimiento las creencias que generalmente son ciertas en un dominio particular.

i) Junto al aumento del valor añadido del conocimiento alcanzado en el proceso de decisión, tanto del problema como de los procedimientos, una resolución efectiva del problema esta orientada hacia la detección de una serie de oportunidades de decisión, pautas de comportamiento o tendencias que se verifiquen con cierta estabilidad y regularidad.

j) Estas oportunidades de decisión son utilizadas en los procesos de negociación, comunicación y búsqueda del consenso entre los actores implicados en el proceso de toma de decisiones, teniendo presente que el establecimiento de caminos de consenso entre los participantes en la resolución del problema es una de las líneas de actuación recomendadas en medio ambiente. 
Como se desprende de lo dicho en las secciones anteriores, las tres aproximaciones consideradas en el trabajo (constructivismo, ciencia postnormal y racionalidad procedimental multicriterio) recogen las anteriores recomendaciones o hechos estilizados que deben caracterizar cualquier metodología utilizada en valoración y selección ambiental. El origen y objetivo de estos tres enfoques 'pragmáticos' es muy similar. Surgen para solventar el carácter simplista y reduccionista del enfoque clásico (racionalidad sustantiva o 'ciencia normal'). Se centran en la mejora de la calidad frente a la búsqueda de la verdad, sugieren la participación de los diferentes actores y la incorporación de sus visiones de la realidad, y contemplan la ambigüedad o incertidumbre asociada a todos los niveles del problema. Por ello, es necesario efectuar un Análisis del Comportamiento del proceso de resolución seguido (Moreno et al., 1998), incluyendo la Validez de la aproximación, la Robustez del modelo y la Estabilidad de las consecuencias. Estas ideas coinciden a groso modo con la propuesta efectuada desde la ciencia postnormal (Functowicz \& Ravetz, 1991, 1994) al hablar de incertidumbre epistemológica, metodológica y técnica.

\section{Conclusiones}

Algunas características de los problemas de selección ambientales como son su complejidad (múltiples actores, criterios, escenarios, etc.), incertidumbre (ausencia de modelos subyacentes e información cierta, lagunas en el conocimiento, etc.), irreversibilidad (existencia de efectos irreversibles con poca verosimilitud) y equidad intergeneracional (consideración de las generaciones futuras) sugieren su resolución utilizando otros enfoques más abiertos, flexibles y realistas que la clásica racionalidad sustantiva.

Junto a los métodos de valoración económica tradicionales, tres de las nuevas aproximaciones propuestas en la línea anterior son tratadas a lo largo de este trabajo: el constructivismo, la ciencia postnormal y el paradigma de la racionalidad procedimental multicriterio. Los aspectos más destacados de estas metodologías se han visto con más detalle a lo largo de la sección 3.

Conforme a los hechos diferenciados o pautas de comportamiento que deben caracterizar cualquier metodología utilizada en valoración y selección ambiental, según se ha visto en la sección 4, cualquiera de las tres aproximaciones consideradas en la sección tercera (constructivismo, ciencia postnormal y racionalidad procedimental multicriterio) está en sintonía, en mayor o menor medida, con las recomendaciones fijadas anteriormente. No obstante, la racionalidad procedimental multicriterio se manifiesta especialmente apropiada si se desea alcanzar una resolución efectiva de los problemas planteados en medio ambiente, debido fundamentalmente a su ontología relativista, su epistemología adaptativa, el constructivismo cognitivo de su metodología, la idoneidad de la modelización seguida y su orientación hacia el aprendizaje, la comprensión, la comunicación y la búsqueda del consenso entre los actores.

\section{Referencias}

(1) Batelle Columbus Lab (1972). Environmental Evaluation System for Water Resource Planning. Springfield.

(2) Beed, C. (1992). Do value judgements affect testing economic theory? Int. J. Soc Econom., 19(2), 6-24. 
(3) Berman, M. (1981): The reenchantment of the World. Cornell University Press, Itacha, N.Y.

(4) Blasco, N.; Moreno, J.M.; Santamaria, R. \& Serrano, C. (1994). Selección de proyectos de inversión publica aislados con impacto ambiental. Documento de trabajo.

(5) Blaug, M. (1992). The methodology of Economics: Or How Economists Explain. Cambridge University Press.

(6) Brugha, C. (1998a). The structure of qualitative decision-making. European Journal of Operational Research, 104, 46-62.

(7) Brugha, C. (1998b). The structure of adjustment decision-making. European Journal of Operational Research, 104, 63-76.

(8) Brugha, C. (1998c). The structure of development decision-making. European Journal of Operational Research, 104, 77-92.

(9) Buchanan, J.T.; Henig, E.J. \& Henig, M.I. (1998). Objectivity and subjectivity in the decision making process. Annals of Operations Research, 80, 333-345.

(10) Cronbach, L. (1975). Beyond the two disciplines of scientific psychology. Am. Psychol, 30, 116-127.

(11) Checkland, P. \& Scholes, J. (1990). Soft System Methodology in Action. Wiley, Chichester.

(12) Ciriacy-Wantrup, S.V. (1952). Resource Conservation: Economics and Policies. Agricultural Experiment Station, Berkeley, University of California Press.

(13) Clawson, M. \& Knetsch, J.L. (1966). Economics of Outdoor Recreation. Johns Hopkins Press, Baltimore.

(14) Davis, R.K. (1963). The Value of Outdoor Recreation: An Economic Study of the Maine Woods. PhD Thesis, Harvard University.

(15) Fishburn, P.C. (1970). Utility Theory for Decision Making. NY, Wiley.

(16) Funtowicz, S.O. \& Ravetz, J.R. (1991). A new scientific methodology for global environmental issues. En: Ecological Economics: The Science and Management of Sustainability [edited by R. Constanza], Columbia University Press, NY, 137-152.

(17) Funtowicz, S.O. \& Ravetz, J.R. (1994). The worth of a songbird: ecological economics as a post-normal science. Ecological Economics, 10(3), 197-207.

(18) Gandy, M. (1996). Crumbling land: the postmodernity debate and the analysis of environmental problems. Prog. Hum. Geogr., 20(1), 23-40.

(19) Geertz, C. (1973). The interpretation of Cultures. Basic Books, N.Y.

(20) Gomez Orea, D. (1988). Evaluación del impacto ambiental de proyectos agrarios. Estudios monográficos n ${ }^{\circ}$ 6. Ministerio de Agricultura, Pesca y Alimentación.

(21) Gomez Orea, D. (1992). Planificación Rural. Ed. Agrícola Española, Madrid.

(22) Griliches, S. (1971). Price Indexes and Quality Change. Studies in New Methods of Measurement. Harvard University Press, Massachusetts.

(23) Hannigan, J.A. (1995). Environmental Sociology: A Social Constructionist Perspective. Routledge. London. 
(24) Hargreaves Heap, S.; Hollis, M.; Lyons, B.; Sugden, R. \& Weale, A. (1992). The Theory of Choice: A Citical Guide. Blackwell, Oxford.

(25) Harvey (1989). The condition of postmodernity: An inquiry into the Origins of Cultural Change. Blakwell, Oxford.

(26) Hausman, D.M. (1992). The inexact and separate science of economics. Cambridge University Press.

(27) Hesse, M. (1980). Revolutions and reconstructions in the philosophy of science. Indiana University Press.

(28) Howard R.A. (1992). Heathens, Heretics and Cults: The Religious Spectrum of Decision Aiding. Interfaces, 22(6), 15-27.

(29) Jacobs, M. (1996). What is socio-ecological economics? Econom. Bull. 1(2), 14-16.

(30) Janssen, R. (1992). Multiobjective decision support for environmental management. Kluwer, Dordrecht.

(31) Kahneman, D. \& Tversky, A. (1979). Prospect theory: an analysis of decision under risk. Econometrica, 47, 263-291.

(32) Keen, P.G.W. \& Scott-Morton. M.S. (1978). Decision Support Systems: an organizational perspective. Addison-Wesley.

(33) Keeney, R. (1992). Value focused thinking. Harvard University Press.

(34) Keeney, R. (1994). Using values in operations research. Operations Research, 36, 369-405.

(35) Keeney, R.L. \& Raiffa, H. (1976). Decisions with Multiple Objectives: Preferences and Value Tradeoffs. Ed. Wiley, New York.

(36) Kuhn, T.S. (1970). The structure of scientific revolution. University of Chicago Press.

(37) Lichtenstein, S. \& Slovic, P. (1971). Reversal of Preferences Between Bids and Choices in Gambling Decisions. J. of Experimental Psychology, 89, 46-55.

(38) Lincoln, Y.S. \& Guba, E.G. (1985). Naturalistic inquiry. Sage Publications, London.

(39) Maturana, H.R. \& Varela, F.J. (1992). The tree of knowledge: the biological roots of human understanding. Shambhala, Boston.

(40) Medawar, P. (1974). Hyothesis and Imagination. En: The filosofy of Karl Popper. Book I [edited by P.A. Schilpp], Open Court Publishing, La Salle, Illinois, 274-291.

(41) Midmore, P. (1996). Towards a postmodern agricultural economics. J. Agric. Econom., 47(1), 1-17.

(42) Mirasgedis, S. \& Diakoulaki, D. (1997). Multicriteria Analysis vs. Externalities Assessment for the Comparative Evaluation of Electricity Generation Systems. European Journal of Operational Research, 102, 364-379.

(43) Moreno-Jiménez, J.M. (1996). Metodología Multicriterio en el Plan Nacional de Regadios (privado).

(44) Moreno-Jimenez, J.M. (1997). Priorización y toma de decisiones ambientales. Actas del ler. Encuentro Iberoamericano de Evaluación y Decisión Multicriterio, 113-145. Santiago de Chile, julio 1997. 
(45) Moreno-Jiménez, J.M. (1998). Una aproximación multicriterio en la selección entre alternativas ambientales. El proceso analítico jerárquico. En: Evaluación Multicriterio: Reflexiones básicas y experiencias en America Latina [edited by Eduardo Martínez \& Mauricio Escudey], capítulo 8, 137-163.

(46) Moreno-Jiménez, J.M.; Aguaron, J.; Cano F. \& Escobar, M.T. (1998). Válidez, Robustez y Estabilidad en Decisión Multicriterio. Análisis de Sensibilidad en el Proceso Analítico Jerárquico. Revista de la Real Academia de Ciencias Exactas, Físicas y Naturales, 92(4), 387-397.

(47) Moreno-Jiménez, J.M.; Aguaron, J.; Escobar, M.T. \& Turon, A. (1999). Multicriteria Procedural Rationality on SISDEMA. European Journal of Operational Research, 119(2), 388-403.

(48) Moreno-Jiménez, J.M. \& Mata, E.J. (1992). Nuevos sistemas informáticos de ayuda a la decisión. Sistemas Decisionales Integrales. Actas de la V Reunión ASEPELT-España, Granada, vol. II, 529-538.

(49) Nelson, J.A. (1996). Feminism, Objectivity and Economics. Routledge, London.

(50) Pulido, A. (1998). Economía Aplicada. Regularidades empíricas y hechos diferenciados. Anales de Economía Aplicada, 1998.

(51) Rosen, S. (1974). Hedonic Prices and Implicit Markets: Product Differenciation in Pure Competition. Journal of Political Economy, 82, 34-55.

(52) Roy, B. (1985). Methodologie Multicritère d'Aide à la Décision. Gestión Económica.

(53) Roy, B. (1993). Decision science or decision-aid science? European Journal of Operational Research, 66, 184-203.

(54) Russell, C.S. (1993). Old lessons and new context in economic-ecological modeling. Resource Policy Consortium, World Bank, Washington, DC.

(55) Saaty, T.L (1977). A scaling method for priorities in herarchical structures. Journal of Mathematical Psychology, 15, 234-281.

(56) Saaty, T.L. (1980). The Analytic Hierarchy Process. McGraw-Hill, New York.

(57) Saaty, T.L. (1994). Fundamentals of Decision Making. RSW Publications.

(58) Saaty, T.L. (1996). The Analytic Network Process. RSW Publications.

(59) Savage, L.J. (1954). The Foundations of Statistics. John Wiley and Sons, New York.

(60) Simon, H.A. (1960). The New Science of Management Decision. Harper \& Row, New York.

(61) Simon, H.A. (1964). Rationality. En: A Dictionary of the Social Science [edited by J. Gould \& W.L. Kolb], The Free Press, Glencoe, IL.

(62) Simon, H.A. (1972). Theories of bounded rationality. En: Decision and Organization [edited by C.B. Radner \& R. Radner], North Holland Publishing Company, Amsterdam.

(63) Suppe, F. (ed.) (1974). The structure of scientific theories. University of Illinois Press, Urbana. 
(64) Tacconi, L. (1998). Scientific methodology for ecological economics. Ecological Economics, 27, 91-105.

(65) Tversky, A. (1988). A rational choice and the framing of decisions. En: Decision making: Descriptive, normative, and prescriptive interactions [edited by D.E. Bell; H. Raiffa, \& A. Tversky], Cambridge University Press.

(66) Tversky, A. \& Kahneman, D. (1972). Judgment under uncertainty: Heuristics and biases. Science, 185, 1124-1131.

(67) Von Newmann, J. \& Morgestern, O. (1944). Theory of Games and Economic Behaviour. Princenton University Press.

(68) Weber, M. (1968). Economy and Society. Bedminster, N.Y.

(69) Woodhill, J. \& Roling, N. (1999). The second wing of the eagle: the human dimension in learning our way to more sustainable future. En: Sustainability Agriculture: Participation, Learning and Action [edited by N. Roling \& Wagemakers], Cambridge University Press. 